\title{
WEIGHTED ESTIMATES FOR CERTAIN ROUGH SINGULAR INTEGRALS
}

\author{
Chunjie Zhang
}

ABSTRACT. In this paper we shall prove some weighted norm inequalities of the form

$$
\int_{R^{n}}|T f(x)|^{p} u(x) d x \leq C_{p} \int_{R^{n}}|f(x)|^{p} N u(x) d x
$$

for certain rough singular integral $T$ and maximal singular integral $T^{*}$. Here $u$ is a nonnegative measurable function on $R^{n}$ and $N$ denotes some maximal operator. As a consequence, some vector valued inequalities for both $T$ and $T^{*}$ are obtained. We shall also get a boundedness result of $T$ on the Triebel-Lizorkin spaces.

\section{Introduction}

It is a classical result for the singular integral

$$
T f(x)=P . V \cdot \int_{R^{n}} K(x-y) f(y) d y
$$

that

$$
\int_{R^{n}}|T f(x)|^{p} u(x) d x \leq C_{p, s} \int_{R^{n}}|f(x)|^{p} M_{s} u(x) d x, 1<p<\infty,
$$

where $M_{s} u=\left(M\left(u^{s}\right)\right)^{1 / s}, s>1$ and $u(x)$ is a nonnegative measurable function on $R^{n}$. The above inequality was first obtained by Cordoba and Fefferman under the condition that $|\hat{K}(\xi)| \leq B$ and $K \in C^{1}\left(R^{n}-\{0\}\right)$, see [8]. Similar result for the Hardy-Littlewood Maximal function $M$ is

$$
\int_{R^{n}}|M f(x)|^{p} u(x) d x \leq C_{p} \int_{R^{n}}|f(x)|^{p} M u(x) d x, 1<p<\infty .
$$

Received December 1, 2006; Revised July 28, 2007.

2000 Mathematics Subject Classification. Primary 42B25.

Key words and phrases. singular integral, weighted norm inequality, vector valued inequality.

Supported partially by NSFC(No. 10601046) and NSFC(No. 10701064). 
Using (1.1) and (1.2), one is ready to get that, for $1<p, r<\infty$

$$
\left\|\left(\sum_{j}\left|T f_{j}\right|^{r}\right)^{1 / r}\right\|_{L^{p}} \leq C_{p, r}\left\|\left(\sum_{j}\left|f_{j}\right|^{r}\right)^{1 / r}\right\|_{L^{p}}
$$

and

$$
\left\|\left(\sum_{j}\left|M f_{j}\right|^{r}\right)^{1 / r}\right\|_{L^{p}} \leq C_{p, r}\left\|\left(\sum_{j}\left|f_{j}\right|^{r}\right)^{1 / r}\right\|_{L^{p}}
$$

by a standard process (see [13], Chapter V). The above estimates (1.2) and (1.4) for $M$ were first proved in [11].

In this paper, we shall consider the rough singular integral defined by

$$
T f(x)=P . V . \int_{R^{n}} \frac{\Omega(y)}{|y|^{n}} f(x-y) d y,
$$

where $\Omega(y)=\Omega\left(y^{\prime}\right) \in L^{1}\left(S^{n-1}\right)$ and $\int \Omega\left(y^{\prime}\right)=0$. Then the Cordoba-Fefferman inequality (1.1) was improved by Hofmann ([15]), where $\Omega\left(y^{\prime}\right)$ was assumed to be in $L^{r}\left(S^{n-1}\right), r>1$. Recall that the maximal function related to $\Omega$ is defined by

$$
M^{\Omega} f(x)=\sup _{r>0} \frac{1}{r^{n}} \int_{|y|<r}\left|\Omega\left(y^{\prime}\right) f(x-y)\right| d y .
$$

Then the theorem of Hofmann can be stated as follow.

Theorem A. Let $T$ and $M_{\Omega}$ be defined as (1.5) and (1.6), where $\Omega \in L^{r}\left(S^{n-1}\right)$, $r>1$ satisfies $\int \Omega\left(y^{\prime}\right)=0$. Then we have

$$
\begin{aligned}
& \int_{R^{n}}|T f(x)|^{p} u(x) d x \leq C \int_{R^{n}}|f(x)|^{p} N_{s} u(x) d x \\
& \int_{R^{n}}\left|M^{\Omega} f(x)\right|^{p} u(x) d x \leq C \int_{R^{n}}|f(x)|^{p} N_{s} u(x) d x .
\end{aligned}
$$

Here $N_{s} u=M_{s} M_{s}^{\Omega} M_{s} u, u(x)$ is a measurable nonnegative function on $R^{n}$ and $s>1$ can be arbitrarily close to 1 .

A better result than Theorem A can be concluded from [9] which says that $T$ and $M^{\Omega}$ are in fact bounded from $L^{p}\left(M_{s} M_{s}^{\tilde{\Omega}^{t}} M_{s} u\right)$ to $L^{p}(u)$. Here $\Omega \in$ $L^{r}\left(S^{n-1}\right), \tilde{\Omega}(x)=\Omega(-x)$ and $t=\max \{0, p(1-r)+r\}$. For $\Omega \in L\left(\ln ^{+} L\right)^{s}, s>$ 1 , Vargas obtained a boundedness of $M^{\Omega}$ from $L^{1}\left(M_{s} u+M^{\tilde{\Omega}\left(\ln ^{+} \tilde{\Omega}\right)^{s}} M_{s} u\right)$ to $W L^{1}(u)$, see [19] where a similar theorem for $T$ was also obtained in $R^{2}$.

Note by [10], or by the classical rotation method in [2], $M^{\Omega} f(x)$ is bounded on $L^{p}\left(R^{n}\right), p>1$ when $\Omega \in L^{1}\left(S^{n-1}\right)$. Therefore, $N_{s} u(x)$ is bounded on $L^{p}\left(R^{n}\right), p>s$ and Theorem A implies the $l^{r}$-valued inequalities for $T$ and $M^{\Omega}$. 
In this paper, we intend to extend Theorem A to more general singular integrals as well as to the maximal singular integral $T^{*}$ defined by

$$
T^{*} f(x)=\sup _{\epsilon>0}\left|T_{\epsilon} f(x)\right|=\sup _{\epsilon>0}\left|\int_{|y|>\epsilon} \frac{\Omega\left(y^{\prime}\right)}{|y|^{n}} f(x-y) d y\right|,
$$

where $\Omega \in L^{1}\left(S^{n-1}\right)$ has mean value zero and satisfies

$$
\sup _{\xi^{\prime} \in S^{n-1}} \int_{S^{n-1}}\left|\Omega\left(y^{\prime}\right)\right|\left(\ln \frac{1}{\left|y^{\prime} \cdot \xi^{\prime}\right|}\right)^{1+\alpha} d y^{\prime} \leq C_{\alpha}
$$

for some $\alpha>0$. The above condition was first introduced by Grafakos and Stefanov ([14]), where the following two theorems were obtained.

Theorem B. Suppose $\Omega \in L^{1}\left(S^{n-1}\right)$ has mean value zero and satisfies (1.8) for some $\alpha>0$. Then the singular integral $T$ defined by (1.5) is bounded on $L^{p}\left(R^{n}\right)$ whenever $(2+\alpha) /(1+\alpha)<p<2+\alpha$.

Theorem C. Suppose $\Omega \in L^{1}\left(S^{n-1}\right)$ has mean value zero and satisfies (1.8) for some $\alpha>1$. Then the maximal singular integral operator $T^{*}$ is bounded on $L^{p}\left(R^{n}\right)$ for $1+3 /(1+2 \alpha)<p<2(2+\alpha) / 3$.

Now we are ready to state our main results.

Theorem 1. Suppose $\Omega \in L^{1}\left(S^{n-1}\right)$ has mean value zero and satisfies condition (1.8) for some $\alpha>0$. Then the singular integral $T$ defined by (1.5) verifies

$$
\int_{R^{n}}|T f(x)|^{p} u d x \leq C_{p, s} \int_{R^{n}}|f(x)|^{p}\left(M_{s} M_{s}^{\Omega} M_{s}+M_{s}\right) u d x
$$

whenever $\lambda(\alpha, s)<p<2+\alpha-\frac{1+\alpha}{s}$ and $s>1+1 / \alpha$ for some $1<\lambda(\alpha, s)<2$.

Theorem 2. Suppose $\Omega \in L^{1}\left(S^{n-1}\right)$ has mean value zero and satisfies condition (1.8) for all $\alpha>0$. Then the maximal singular integral operator $T^{*}$ verifies

$$
\int_{R^{n}}\left|T^{*} f(x)\right|^{p} u d x \leq C_{p, s} \int_{R^{n}}|f(x)|^{p}\left(M_{s} M_{s}^{\Omega} M_{s}+M_{s}\right) u d x
$$

for $1<p<\infty$ and any $s>1$.

Our proof of Theorem 1 follows essentially the proof of Theorem A, but keeps track of the constants in each step in order to get the precise range of the index $p$. We shall see that if (1.8) holds for all $\alpha>0$, then Theorem 1 is valid for all $1<p<\infty$. Note by [14], the condition of Theorem 1 contains the case $\Omega \in L^{r}\left(S^{n-1}\right), r>1$. Also we have the fact that $M_{s} u(x) \leq\|\Omega\|_{L^{r}}^{-1} M^{\Omega} M_{s} u(x)$ almost everywhere. Thus Theorem 1 is an extension of Theorem A. In Theorem 2, we have assumed (1.8) for all $\alpha>0$ rather than some fixed $\alpha$ in order to avoid heavy computation. However, if we merely consider the $L^{p}$-boundedness of $T^{*}$, then our proof will provide a wider range of $p$ than that of Theorem $\mathrm{C}$. 


\section{Some preparation}

Let $T f(x)$ be defined by (1.5) and we decompose it as

$$
T f(x)=\sum_{k=-\infty}^{+\infty} \sigma_{k} * f(x),
$$

where

$$
\sigma_{k}(x)=\frac{\Omega\left(x^{\prime}\right)}{|x|^{n}} \chi_{\left\{2^{k}<|x| \leq 2^{k+1}\right\}} .
$$

It is trivial that $\int_{R^{n}} \sigma_{k}(x) d x=0$ and $\left\|\sigma_{k}(x)\right\|_{L^{1}} \leq C\left\|\Omega\left(x^{\prime}\right)\right\|_{L^{1}}$ for all $k \in Z$. We also denote $\tilde{\sigma}_{k}(x)=\sigma_{k}(-x)$. But since it always has the same properties with $\sigma_{k}(x)$, we shall abuse the notation and simply write $\sigma_{k}$ in either case.

Lemma 1. Let $\Omega \in L^{1}\left(S^{n-1}\right)$ satisfy $\int \Omega\left(y^{\prime}\right) d \sigma\left(y^{\prime}\right)=0$ and condition (1.8) for some $\alpha>0$. Then we have the following estimate for $\left\{\sigma_{k}\right\}$,

$$
\left|\hat{\sigma}_{k}(\xi)\right| \leq C \min \left\{\left|2^{k} \xi\right|,\left(\ln \left|2^{k} \xi\right|\right)^{-(1+\alpha)}\right\}, k \in Z .
$$

One may find the above conclusion in [14] or compute directly from (1.8). Next we define

$$
M^{\sigma} f(x)=\sup _{k}\left|\sigma_{k}\right| *|f|(x) .
$$

It is not hard to check that $M^{\sigma}$ is equivalent to $M^{\Omega}$ defined by (1.6). But it will be convenient to use $M^{\sigma}$ in our context. The following lemma is stated with $M^{\sigma}$ and was obtained in the proof of Theorem 1 in [14].

Lemma 2. Let $\Omega$ be as in Lemma 1. Then $M^{\sigma}$ is bounded on $L^{p}\left(R^{n}\right)$ for $(2+\alpha) /(1+\alpha)<p<2+\alpha$.

Taking a nonnegative function $\Phi(t) \in C_{c}^{\infty}\left(R^{+}\right)$such that $\sum_{j=-\infty}^{+\infty} \Phi^{3}\left(2^{j} t\right)=$ 1, we define $S_{j} f(x)=\Psi_{j} * f(x)$, where $\hat{\Psi}_{j}(\xi)=\Phi\left(2^{j}|\xi|\right)$. It is then a classical result that the Littlewood-Paley $g$-function

$$
g(f)=\left(\sum_{j=-\infty}^{+\infty}\left|S_{j} f\right|^{2}\right)^{1 / 2}
$$

satisfies

$$
\|g(f)\|_{L^{p}(w)} \leq C_{p, w}\|f\|_{L^{p}(w)}, 1<p<\infty, w \in A_{p}
$$

and

$$
\left\|\sum_{j} S_{j} f_{j}\right\|_{L^{p}(w)} \leq C_{p, w}\left\|\left(\sum_{j}\left|f_{j}\right|^{2}\right)^{1 / 2}\right\|_{L^{p}(w)}, 1<p<\infty, w \in A_{p} .
$$

In fact, (2.2) was obtained by viewing $g(f)$ as an $l^{2}$-valued singular integral (see [16]). The second inequality is an easy consequence of the first one by duality. 
Lemma 3. Let $\sigma_{k}$ be as in Lemma 1. Then for any $s>1$, we have

$$
\left\|\left(\sum_{k}\left|\sigma_{k} * g_{k}\right|^{2}\right)^{1 / 2}\right\|_{L^{q}(u)} \leq C_{q, s}\left\|\left(\sum_{k}\left|g_{k}\right|^{2}\right)^{1 / 2}\right\|_{L^{q}\left(M_{s}^{\sigma} u\right)},
$$

where $2 \leq q<4+2 \alpha-\frac{2(1+\alpha)}{s}$ and $u$ is nonnegative measurable on $R^{n}$.

Proof. The case $q=2$ is trivial. When $q>2$, by duality we get a $v \in L^{\left(\frac{q}{2}\right)^{\prime}}(u)$ with unit norm such that

$$
\left\|\left(\sum_{k}\left|\sigma_{k} * g_{k}\right|^{2}\right)^{1 / 2}\right\|_{L^{q}(u)}^{2}=\int_{R^{n}} \sum_{k}\left|\sigma_{k} * g_{k}\right|^{2} \cdot v(x) u(x) d x .
$$

Since $\left\|\sigma_{k}\right\|_{L^{1}} \leq C$, the above expression does not exceed

$$
\sum_{k} \int_{R^{n}}\left|\sigma_{k}\right| *\left|g_{k}\right|^{2} v(x) u(x) d x=\sum_{k} \int_{R^{n}}\left|g_{k}\right|^{2}\left|\sigma_{k}\right| *(v \cdot u)(x) d x .
$$

Fix $s>1$ and let $r=q s / 2$. By Hölder's inequality, we have

$$
\left|\sigma_{k}\right| *(v \cdot u)=\left(\left|\sigma_{k}\right| * u^{2 r / q}\right)^{\frac{1}{r}} \cdot\left(\left|\sigma_{k}\right| *\left(v^{r^{\prime}} u^{r^{\prime} /(q / 2)^{\prime}}\right)\right)^{\frac{1}{r^{\prime}}} .
$$

Again by Hölder's inequality with exponents $q / 2$ and $(q / 2)^{\prime}$,

$$
\begin{aligned}
& \sum_{k} \int_{R^{n}}\left|g_{k}\right|^{2} \sigma_{k} *(v \cdot u) d x \\
\leq & \int_{R^{n}}\left(\sum_{k}\left|g_{k}\right|^{2}\right)\left(M^{\sigma} u^{s}\right)^{1 / r} \cdot M^{\sigma}\left(v^{r^{\prime}} u^{r^{\prime} /(q / 2)^{\prime}}\right)^{\frac{1}{r^{\prime}}} d x \\
\leq & \left\|\left(\sum_{k}\left|g_{k}\right|^{2}\right)^{1 / 2}\right\|_{L^{q}\left(M_{s}^{\sigma} u\right)}^{2} \cdot\left(\int_{R^{n}}\left(M^{\sigma}\left(v^{r^{\prime}} u^{r^{\prime} /(q / 2)^{\prime}}\right)\right)^{(q / 2)^{\prime} / r^{\prime}} d x\right)^{\frac{1}{(q / 2)^{\prime}}} .
\end{aligned}
$$

If we let $\left(\frac{q}{2}\right)^{\prime} / r^{\prime} \in\left(\frac{2+\alpha}{1+\alpha}, 2+\alpha\right)$, then Lemma 2 is applied to get

$$
\int_{R^{n}}\left(M^{\sigma}\left(v^{r^{\prime}} u^{r^{\prime} /(q / 2)^{\prime}}\right)\right)^{(q / 2)^{\prime} / r^{\prime}} \leq \int_{R^{n}} v^{(q / 2)^{\prime}} u d x=1 .
$$

Remembering $s=2 r / q$ and the requirement that

$$
\frac{2+\alpha}{1+\alpha}<\frac{(q / 2)^{\prime}}{r^{\prime}}<2+\alpha
$$

we conclude

$$
\frac{2(s-1)}{s(1+\alpha)}+2<q<4+2 \alpha-\frac{2(1+\alpha)}{s} .
$$

The left side of the above inequality is larger than 2. But this in fact gives no restriction since we can interpolate between the case $q=2$ and some large $q$ satisfying (2.4). 


\section{Proof of the theorems}

Proof of Theorem 1. We first consider the case $p \geq 2$. Since $M_{s} u \in A_{1}$ (see [7]) and $u \leq M_{s} u$, we can restrict ourselves to show

$$
\int_{R^{n}}|T f(x)|^{p} w(x) d x \leq C \int_{R^{n}}|f(x)|^{p} M_{s} M_{s}^{\sigma} w(x) d x
$$

with $w(x) \in A_{1}$. Using the notations stated after Lemma 2, we decompose $T f(x)$ into

$$
\sum_{k} \sum_{j} S_{j+k}^{3}\left(\sigma_{k} * f\right)=\sum_{j} \sum_{k} S_{j+k}^{3}\left(\sigma_{k} * f\right)=\sum_{j} T_{j} f .
$$

Then by Minkowski's inequality, it suffices to show

$$
\left\|T_{j} f\right\|_{L^{p}(w)} \leq C(1+|j|)^{-\gamma(\alpha, p, s)}\|f\|_{L^{p}\left(M_{s} M_{s}^{\sigma} w\right)},
$$

where $\gamma(\alpha, p, s)$ should be strictly larger than 1 in order to be summable. Thus the range of $p$ depending on $\alpha$ and $s$ will be obtained.

To prove (3.1), by interpolation, we only have to show

$$
\left\|T_{j} f\right\|_{L^{2}(w)} \leq C(1+|j|)^{-\gamma_{1}(\alpha, s)}\|f\|_{L^{2}\left(M_{s} M_{s}^{\sigma} w\right)}
$$

and

$$
\left\|T_{j} f\right\|_{L^{p}(w)} \leq C\|f\|_{L^{p}\left(M_{s} M_{s}^{\sigma} w\right)} .
$$

Inequality (3.3) is immediate by Lemma 3 since

$$
\begin{aligned}
\left\|T_{j} f\right\|_{L^{q}(w)} & =\left\|\sum_{k} S_{j+k}^{3} \sigma_{k} * f\right\|_{L^{q}(w)} \leq C\left\|\left(\sum_{k}\left|S_{j+k}^{2} \sigma_{k} * f\right|^{2}\right)^{\frac{1}{2}}\right\|_{L^{q}(w)} \\
& \leq C\left\|\left(\sum_{k}\left|S_{j+k}^{2} f\right|^{2}\right)^{\frac{1}{2}}\right\|_{L^{q}\left(M_{s} M_{s}^{\sigma} w\right)} \leq C\|f\|_{L^{q}\left(M_{s} M_{s}^{\sigma} w\right)} .
\end{aligned}
$$

Here we have also used (2.3) in the first inequality and (2.2) in the last inequality. However, we have to pay special attention to (3.3) because it holds only for $2 \leq q<4+2 \alpha-\frac{2(1+\alpha)}{s}$. Next we turn to (3.2). By (2.3),

$$
\left\|T_{j} f\right\|_{L^{2}(w)}^{2}=\left\|\sum_{k} S_{j+k}^{3} \sigma_{k} * f\right\|_{L^{2}(w)}^{2} \leq \sum_{k} \int_{R^{n}}\left|S_{j+k}^{2} \sigma_{k} * f\right|^{2} w(x) d x .
$$

Therefore, (3.2) will be proved if we show

$$
\int_{R^{n}}\left|\sigma_{k} * S_{j+k} f\right|^{2} w(x) d x \leq C(1+|j|)^{-2 \gamma_{1}(\alpha, s)} \int_{R^{n}}|h|^{2} M_{s} M_{s}^{\sigma} w(x) d x
$$

because we are able to substitute $S_{j+k} f$ for $h$ and apply (2.2) once more. 
Now it remains to show (3.4). But this just follows by another interpolation with change of measures (see [17] or [1], p. 115) between

$$
\int_{R^{n}}\left|\sigma_{k} * S_{j+k} h\right|^{2} d x \leq C(1+|j|)^{-2 \gamma_{2}(\alpha)} \int_{R^{n}}|h|^{2} d x
$$

and

$$
\int_{R^{n}}\left|\sigma_{k} * S_{j+k} h\right|^{2} w^{s} d x \leq C \int_{R^{n}}|h|^{2} M M^{\sigma}\left(w^{s}\right) d x .
$$

Since $\left\|\sigma_{k}\right\|_{L^{1}} \leq C$ and $\left\|\Psi_{j+k}\right\|_{L^{1}} \leq C$, the left side of (3.6) does not exceed

$$
\int_{R^{n}}\left|\sigma_{k}\right| *\left|S_{j+k}\right| *|h|^{2} w^{s} d x
$$

and (3.6) then follows by duality. Using Plancherel's theorem, the left side of (3.5) equals

$$
\int_{R^{n}}\left|\hat{\sigma}_{k}\right|^{2} \Phi_{j+k}^{2}(\xi)|\hat{h}|^{2} d \xi
$$

which by Lemma 1 is no larger than

$$
\begin{aligned}
& C \int_{2^{-j-k-1}<|\xi| \leq 2^{-j-k}} \min \left\{2^{k}|\xi|,\left(\ln \left|2^{k} \xi\right|\right)^{-1-\alpha}\right\}^{2}|\hat{h}|^{2} d \xi \\
\leq & C(1+|j|)^{-2(1+\alpha)}\|h\|_{L^{2}}^{2} .
\end{aligned}
$$

Thus we have proved (3.5) with $\gamma_{2}=1+\alpha$. By interpolation, we find that $\gamma_{1}(\alpha, s)=(1+\alpha)(1-1 / s)$. Since it must be larger than 1 , we have to assume $s>1+1 / \alpha$. The constant $\gamma(\alpha, p, s)$ is also obtained by interpolation so that $\gamma(\alpha, p, s)=\theta \gamma_{1}(\alpha, s)$ where $\theta$ satisfies $\frac{\theta}{2}+\frac{1-\theta}{q}=\frac{1}{p}$ and $q \in(2,4+2 \alpha-$ $2(1+\alpha) / s)$. Again we have to let $\gamma(\alpha, p, s)>1$ and finally conclude $2 \leq p<$ $2+\alpha-\frac{1+\alpha}{s}$.

Next we consider the case $p<2$. In [15], the conclusion of our Lemma 3 here was stated for all $1<q<\infty$. But Watson found that the proof there when $1<q<2$ was incorrect and a correction was written by Hofmann and Watson to fix this problem, see the appendix. We shall sketch its idea briefly to see that it adapts to our situation here. Note we have to show

$$
\int_{R^{n}}|T f|^{p} u d x \leq C_{p, s} \int_{R^{n}}|f|^{p}\left(M_{s} M_{s}^{\sigma} M_{s}+M_{s}\right) u d x
$$

for $\lambda(\alpha, s)<p<2$. And it is enough to prove

$$
\int_{R^{n}}|T f|^{p} v^{1 / r} \leq C_{p, s} \int_{R^{n}}|f|^{p}\left(M M^{\sigma} M_{s} v+M_{s} v\right)^{1 / r}
$$

for all $r, s>1$.

First it was shown in [15] that

$$
\int_{R^{n}}\left|M^{\sigma} f\right|^{p} v^{1 / r} \leq C_{p, r} \int_{R^{n}}|f|^{p}\left(M M^{\sigma} M_{s} v+M_{s} v\right)^{1 / r}
$$


always holds with the same exponents as (3.7). Then by Lemma 2,

$$
\int_{R^{n}}\left(\sup _{k}\left|\sigma_{k} * f_{k}\right|\right)^{p} d x \leq C_{p} \int_{R^{n}}\left(\sup _{k}\left|f_{k}\right|\right)^{p} d x, \frac{2+\alpha}{1+\alpha}<p<2
$$

since $\sup _{k}\left|\sigma_{k} * f_{k}\right| \leq M^{\sigma}\left(\sup _{k}\left|f_{k}\right|\right)$. On the other hand, as in the appendix, we can show

$$
\int_{R^{n}} \sum_{k}\left|\sigma_{k} * f_{k}\right|^{p} v \leq C_{p} \int_{R^{n}} \sum_{k}\left|f_{k}\right|^{p}\left(M M^{\sigma} M_{s} v+M_{s} v\right), 1<p<2 .
$$

Interpolating with change of measures between (3.9) and (3.10) gives

$$
\int_{R^{n}}\left(\sum_{k}\left|\sigma_{k} * f_{k}\right|^{2}\right)^{p / 2} v^{1 / r_{1}} \leq C_{p, r_{1}} \int_{R^{n}}\left(\sum_{k}\left|f_{k}\right|^{2}\right)^{p / 2}\left(M M^{\sigma} M_{s} v+M_{s} v\right)^{1 / r_{1}}
$$

which is the substitute of Lemma 3 when $p<2$. Following the same argument for $p>2$, we get (3.7) thus (3.8) which yields

$$
\int_{R^{n}}\left(\sup _{k}\left|\sigma_{k} * f_{k}\right|\right)^{p} v^{1 / r_{1}} \leq C_{p, r_{1}} \int_{R^{n}}\left(\sup _{k}\left|f_{k}\right|\right)^{p}\left(M M^{\sigma} M_{s} v+M_{s} v\right)^{1 / r_{1}}
$$

with $\lambda_{1}\left(\alpha, s, r_{1}\right)<p<2$. We proceed to interpolate between $\left(3.9^{\prime}\right)$ and $(3.10)$. By induction we get a sequence $\left\{r_{k}\right\}$ which tends to 1 . Thus for any fixed $r, s>$ 1 , we stop at some $r_{k_{0}}<r$ and derive the desired range $\lambda_{k_{0}}\left(\alpha, s, r_{k_{0}}\right)<p<2$. It should be complicated to give the explicit expression of $\lambda_{k_{0}}$. However, it goes to 1 when $\alpha$ tends to $\infty$.

Remark 1. Taking $v=u^{r}$ in (3.8) and relabelling the exponents, we get

$$
\int_{R^{n}}\left(M^{\sigma} f\right)^{p} u(x) d x \leq C_{p, s} \int_{R^{n}}|f|^{p}\left(M_{s} M_{s}^{\sigma} M_{s}+M_{s}\right) u(x) d x .
$$

If (1.8) is satisfied for all $\alpha>0$, then (3.11) holds for all $1<p<\infty$.

Proof of theorem 2. We shall continuously apply Theorem 1 and the remark above. Now that (1.8) holds for all $\alpha>0,(1.9)$ and (3.11) are valid for all $1<p<\infty$.

Let us first note that

$$
T_{\epsilon} f(x)=\int_{\epsilon<|y| \leq 2^{k}} \frac{\Omega\left(y^{\prime}\right)}{|y|^{n}} f(x-y) d y+\sum_{j=k_{0}}^{+\infty} \sigma_{j} * f(x)
$$

for some $k_{0}$ satisfying $2^{k_{0}-1} \leq \epsilon<2^{k_{0}}$. Therefore

$$
T^{*} f(x) \leq M^{\sigma} f(x)+\sup _{k}\left|\sum_{j=k}^{+\infty} \sigma_{j} * f(x)\right| .
$$


By Remark 1, we obtain the required estimate for $M^{\sigma}$. Taking a $C^{\infty}$ function $\phi$ which is supported on $B(0,2)$ and equals to 1 on $B(0,1)$, and setting $\hat{\psi}_{k}(\xi)=$ $\phi\left(2^{k} \xi\right)$, we have

$$
\begin{aligned}
\sum_{j=k}^{+\infty} \sigma_{j} * f & =\psi_{k} * T f-\psi_{k} * \sum_{j=-\infty}^{k-1} \sigma_{j} * f+\left(\delta-\phi_{k}\right) * \sum_{j=k}^{+\infty} \sigma_{j} * f \\
& =I_{1}+I_{2}+I_{3}
\end{aligned}
$$

where $\delta$ is the dirac measure at zero. Obviously $\sup _{k} I_{1} \leq M(T f)(x)$ and $\sup _{k} I_{k} \leq M f(x)$ (see [10], p. 348). The required estimates for those two terms are obtained by applying Theorem 1 .

Now we turn to

$$
\sup _{k} I_{3}=\sup _{k}\left|\left(\delta-\psi_{k}\right) * \sum_{j=k}^{\infty} \sigma_{j} * f\right|
$$

which is bounded by

$$
\sum_{j=0}^{\infty} \sup _{k}\left|\left(\delta-\psi_{k}\right) * \sigma_{j+k} * f\right|=\sum_{j=0}^{+\infty} K_{j} f(x) .
$$

For any nonnegative measurable function $u$,

$$
\begin{aligned}
\int\left(\sup _{k}\left|\left(\delta-\psi_{k}\right) * \sigma_{j+k} * f\right|\right)^{q} u d x & \leq 2^{q} \int\left(\sup _{k} M\left(\sigma_{j+k} * f\right)\right)^{q} u d x \\
& \leq 2^{q} \int\left(M\left(\sup _{k}\left|\sigma_{j+k} * f\right|\right)\right)^{q} u d x \\
& \leq 2^{q} \int\left(M\left(M^{\sigma} f \mid\right)\right)^{q} u d x .
\end{aligned}
$$

By Remark 1, we get

$$
\int_{R^{n}}\left|K_{j} f(x)\right|^{q} u(x) d x \leq C \int_{R^{n}}|f(x)|^{q} N_{s} u(x) d x .
$$

When $q=2$, substituting $u^{s}$ for $u$ we get

$$
\int_{R^{n}}\left|K_{j} f(x)\right|^{2} u^{s}(x) d x \leq C \int_{R^{n}} \mid f(x)^{2} N_{s}\left(u^{s}\right)(x) d x .
$$

Replacing $s$ with $s^{2}$ in (3.12) gives

$$
\int_{R^{n}}\left|K_{j} f(x)\right|^{q} u(x) d x \leq C \int_{R^{n}}|f(x)|^{q} N_{s^{2}} u(x) d x .
$$

Later we shall prove, by Plancherel's theorem that

$$
\int_{R^{n}}\left|K_{j} f(x)\right|^{2} d x \leq(1+j)^{-2(1 / 2+\alpha)} \int_{R^{n}}|f(x)|^{2} d x, \forall \alpha>0 .
$$


Interpolating with change of measures between (3.13) and (3.15) gives

$$
\int_{R^{n}}\left|K_{j} f(x)\right|^{2} u(x) d x \leq C(1+j)^{-2 \gamma_{3}(\alpha, s)} \int_{R^{n}}|f(x)|^{2} N_{s^{2}} u(x) d x,
$$

where $\gamma_{3}(\alpha, s)=(1 / 2+\alpha)(1-1 / s)$. By another interpolation between $(3.14)$ and (3.16), we get

$$
\int_{R^{n}}\left|K_{j} f(x)\right|^{p} u(x) d x \leq C(1+j)^{-2 \gamma_{4}(\alpha, s, p)} \int_{R^{n}}|f(x)|^{p} N_{s^{2}} u(x) d x .
$$

Replacing $s^{2}$ with $s$ in the above inequality and taking $\alpha$ sufficiently large so that $\gamma_{4}>1$, we obtain the required estimate for $\sup _{k} I_{3}$ by taking sums over $j \geq 0$.

Finally let us look at (3.15). It is easy to see that

$$
K_{j} f(x) \leq\left(\sum_{k}\left|\left(\delta-\psi_{k}\right) * \sigma_{j+k} * f\right|^{2}\right)^{1 / 2} .
$$

Thus by Plancherel's theorem,

$$
\begin{aligned}
\int_{R^{n}}\left|K_{j} f(x)\right|^{2} d x & \leq \sum_{k} \int_{R^{n}}\left|\left(\delta-\psi_{k}\right) * \sigma_{j+k} * f\right|^{2} d x \\
& =\sum_{k} \int_{R^{n}}\left|1-\phi\left(2^{k} \xi\right)\right|^{2}\left|\hat{\sigma}_{j+k}(\xi)\right|^{2}|\hat{f}|^{2} d \xi \\
& =\sum_{k} \frac{\chi_{\left|2^{k} \xi\right|>2}(\xi)}{\left(\ln \left|2^{j+k} \xi\right|\right)^{2(1+\alpha)}} \int_{R^{n}}|\hat{f}|^{2} d \xi \\
& =\sum_{k} \frac{C \chi_{\left|2^{k} \xi\right|>2}(\xi)}{\left(j+\ln \left|2^{k} \xi\right|\right)^{2(1+\alpha)}} \int_{R^{n}}|f(x)|^{2} d x \\
& =C(1+j)^{-1-2 \alpha}\|f\|_{L^{2}}^{2} .
\end{aligned}
$$

Remark 2. Note that (3.15) is more precise than the corresponding $L^{2}$-estimate in [14]. Thus by plugging it into the proof of Theorem C, we shall get a better range of $p$ and also a weaker restriction on $\alpha(\alpha>1 / 2$ is enough to guarantee the $L^{p}$ boundedness of $\left.T^{*}\right)$.

\section{Applications}

We all know the close connection between the weighted norm inequality and the $l^{r}$-valued inequality. With Theorem 1 in hand, we are ready to obtain the following corollary. 
Corollary 1. Let $\Omega$ have mean value zero and satisfy (1.8) for all $\alpha>0$. Then the singular integral $T$ verifies

$$
\left\|\left(\sum_{k}\left|T f_{k}\right|^{r}\right)^{1 / r}\right\|_{L^{p}} \leq C_{r, p}\left\|\left(\sum_{k}\left|f_{k}\right|^{r}\right)^{1 / r}\right\|_{L^{p}}
$$

for all $1<r, p<\infty$. Similar result holds for $M^{\sigma}$.

The proof of (4.1) when $p \geq r$ is direct while the case $1<p<r$ follows by duality. Similar estimate for $M^{\sigma}$ can be reached by viewing it as a linearizable operator described in [13], Chapter V. However, the $l^{r}$-valued inequality for $T^{*}$ is a bit complicated since it can not be treated like $T$ or $M^{\sigma}$. One may follow the method in [20] to prove a weighted norm inequality

$$
\int_{R^{n}}\left|T^{*} f(x)\right|^{2} w(x) d x \leq C \int_{R^{n}}|f(x)|^{2} w(x) d x,
$$

where $w$ belongs to some special kind of weights $A_{2}^{\Omega}$, and then use an extrapolation property of $A_{p}^{\Omega}$ to proceed.

A further application comes from the relationship between the $l^{r}$-valued inequality and the boundedness of linear operators on Triebel-Lizorkin spaces. Let us recall the definition of homogeneous Triebel-Lizorkin space $\dot{F}_{p}^{\beta, r}$. Take $\Phi \in C_{c}^{\infty}\left(R^{n}\right)$ which satisfies $\operatorname{supp}(f) \subset\{\xi: 1 / 2<|\xi|<2\}$ and $\Phi(\xi) \geq 1$ if $3 / 5<|\xi|<5 / 3$. Define $\Psi_{k}(x)$ by $\hat{\Psi}_{k}(\xi)=\Phi\left(2^{k} \xi\right)$. Then we say a tempered distribution $f$ belongs to $\dot{F}_{p}^{\beta, r}, \beta \in R, 1<p, r<\infty$ if and only if

$$
\|f\|_{\dot{F}_{p}^{\beta, r}}=\left\|\left(\sum_{k}\left|2^{-k \beta} \Psi_{k} * f\right|^{r}\right)^{1 / r}\right\|_{L^{p}}<+\infty .
$$

From [12] we know that $\dot{F}_{p}^{0,2}=H^{p}$ when $0<p \leq 1, \dot{F}_{p}^{0,2}=L^{p}$ when $1<p<\infty$ and $\dot{F}_{p}^{\beta, 2}=\dot{L}_{\beta}^{p}, p>1$ is the Sobolev space.

The boundedness of the singular integrals on $\dot{F}_{p}^{\beta, r}$ has been studied by many authors (see $[3,4,6]$ ). Following is one of the theorems taken from [3].

Theorem D. Suppose $\Omega \in L^{q}\left(S^{n-1}\right), q>1$ satisfies $\int \Omega\left(y^{\prime}\right)=0$. Then the singular integral $T$ defined by (1.5) is bounded on $\dot{F}_{p}^{\beta, r}, \beta \in R, 1<p, r<\infty$.

Now by applying (4.1), we can extend the above theorem to (see [5]).

Corollary 2. Suppose $\Omega$ satisfies (1.8) uniformly for all $\alpha>0$ and has mean value zero. Then the singular integral defined by (1.5) is bounded on $\dot{F}_{p}^{\beta, r}, \beta>$ $0,1<r, p<\infty$. 
Proof. Since $T$ commutes with convolution,

$$
\begin{aligned}
\|T f\|_{\dot{F}_{p}^{\beta, r}} & =\left\|\left(\sum_{k}\left|2^{-k \beta} \Psi_{k} * T f\right|^{r}\right)^{1 / r}\right\|_{L^{p}} \\
& =\left\|\left(\sum_{k}\left|T\left(2^{-k \beta} \Psi_{k} * f\right)\right|^{r}\right)^{1 / r}\right\|_{L^{p}} \\
& \leq C_{p, r}\left\|\left(\sum_{k}\left|2^{-k \beta} \Psi_{k} * f\right|^{r}\right)^{1 / r}\right\|_{L^{p}} \\
& =C_{p, r}\|f\|_{\dot{F}_{p}^{\beta, r}} .
\end{aligned}
$$

Here (4.1) is applied with $f_{k}$ being replaced by $2^{-k \beta} \Psi_{k} * f$.

Acknowledgement. The author would like to express his appreciation to the referee for his good suggestion and for providing the Erratum which is listed below as the appendix.

\title{
Appendix
}

Erratum to Steve Hofmann's paper, "Weighted norm inequalities and vector-valued inequalities for certain rough operators"

\author{
Steve Hofmann and David K. Watson \\ September 1994
}

In [15], the first-named author, following J. Duoandikoetxea and J. L. Rubio de Francia [10], studies singular integrals and maximal functions on $R^{n}$ of the form

$$
\begin{aligned}
& T^{\sigma} f=\sum_{k=-\infty}^{\infty} \sigma_{k} * f, \\
& M^{\mu} f=\sup _{k \in Z}\left|\mu_{k} * f\right|,
\end{aligned}
$$

with $\left\{\sigma_{k}\right\}_{k=-\infty}^{\infty}$ and $\left\{\mu_{k}\right\}_{k=-\infty}^{\infty}$ being sequence of Borel measures such that $\int d \sigma_{k}=0, \mu_{k} \geq 0$, and $\left\|\sigma_{k}\right\| \leq 1,\left\|\mu_{k}\right\| \leq 1$. In addition, these measures are assumed to satisfy mild orthogonality generalized smoothness properties, as embodied by certain Fourier transform estimates $[15,(1.9)$ and (1.10)]. These hypotheses were seen in [10] to be sufficient to show that $T^{\sigma}$ and $M^{\mu}$ are bounded on $L^{p}$ for all $p>1$. 
The main result of [15], Theorem 1.11, states that for $1<p<\infty$, for all $t>1$, and for all nonnegative locally integrable $u$, the inequalities

$$
\begin{aligned}
& \int\left|T^{\sigma} f\right|^{p} u \leq C_{p, t} \int|f|^{p} M_{t} M_{t}^{|\widetilde{\sigma}|} M_{t} u, \\
& \int\left|M^{\mu} f\right|^{p} u \leq C_{p, t} \int|f|^{p} M_{t} M_{t}^{|\widetilde{\mu}|} M_{t} u,
\end{aligned}
$$

hold with constant independent of $u$. Here $M$ is the Hardy-Littlewood Maximal operator, $M_{t} f=\left(M\left(|f|^{t}\right)\right)^{1 / t}$, and $\widetilde{\mu}_{k}$ is defined by

$$
\int f(x) d \widetilde{\mu}_{k}=\int f(-x) d \mu_{k}
$$

From estimates of the sort appearing in this theorem, it is well-known that vector-valued inequalities follow $[15,(1.2)]$.

In [15], the proof of the theorem depends on a lemma, [15, Lemma 2.9, p. 8], which states that the inequality

$$
\left\|\left(\sum_{k}\left|\sigma_{k} * g_{k}\right|^{2}\right)^{1 / 2}\right\|_{p, w} \leq C_{p, w}\left\|\left(\sum_{k}\left|g_{k}\right|^{2}\right)^{1 / 2}\right\|_{p, M_{s}^{|\widetilde{\sigma}|} w}
$$

holds for $1<p<\infty$ and for $w \in A_{1}$, with constant depending only on $p$ and the $A_{1}$ constant of $w$. The proof of the inequality in [15] is correct only for the case $p \geq 2$; for the case $1<p<2$ the proof given is incorrect because an assumption of positivity is inconsistent with the use of random variables. In this note we prove a substitute inequality (see (S) below) and show how this new inequality still yields the theorem. This is done by combining the essential strategy of [15] with some additional considerations. The error in [15] was found by the second author, who also suggested the corrected proof, given here.

In [15], the theorem at a given exponent $p$ follows from the use of $(\dagger)$ at the same exponent, so we must prove the theorem for the case $1<p<2$. To do so, it will suffice to prove the following two inequalities:

$$
\begin{gathered}
\int\left|T^{\sigma} f\right|^{p} v^{1 / r} \leq C_{p, r} \int|f|^{p}\left(M M^{|\widetilde{\sigma}|} M_{s} v\right)^{1 / r} \\
\int\left|M^{\mu} f\right|^{p} v^{1 / r} \leq C_{p, r} \int|f|^{p}\left(M M^{|\widetilde{\mu}|} M_{s} v\right)^{1 / r}
\end{gathered}
$$

for all locally integrable $v$ and all $r, s>1$. To see that these inequalities will give the desired conclusions, substitute $v^{r}$ for $v$ in $(*)$. With this substitution, the weight on the left becomes $v$ and the weight on the right becomes $M_{r} M_{r}^{\widetilde{\mu}} M_{r s} v$, which can then be replaced with the weight $M_{t} M_{t}^{\widetilde{\mu}} M_{t} v$ for $t=r s$. This replacement works because the new weight is pointwise larger, as may be seen using Hölder's inequality with exponents $s$ for the first two maximal operators in the original weight. But this gives the result that we want to show, since $t$ 
may be chosen as close to 1 as is wished, and similar substitutions will work for $M^{\mu}$.

The proof of $(*),(* *)$ follow by a bootstrapping argument from the following result.

Lemma. Let $1<p<2$, and suppose the inequality

$$
\int\left(M^{|\sigma|} f\right)^{p} v^{1 / 3} \leq C_{p, r} \int|f|^{p}\left(M M^{|\widetilde{\sigma}|} M_{s} v\right)^{1 / r}
$$

holds for some exponents $r=r_{0}>1$, and $s=\bar{s}>1$. Then (*) also holds for all $r>r_{1}$ and $s>\bar{s}$, where $\frac{1}{r_{1}}=\frac{1}{r_{0}}+\frac{p}{2}\left(1-\frac{1}{r_{0}}\right)$.

Since we know the unweighted bounds for $M^{|\sigma|}$, we know $(* * *)$ for $r_{0}=\infty$ and any $\bar{s}>1$. The lemma then gives $(*)$ for $r>r_{1}=2 / p$ and $s>\bar{s}$, and the reduction in [15] of the maximal operator inequalities to the singular integral operator inequalities then gives $(* *)$ and $(* * *)$ for the same range of exponents. We can now apply $(* * *)$ in the lemma with $r>r_{1}$ in place of $r_{0}$ to increase the range of $r$ even more. Fixing $s>1$ and choosing a sequence of values for $\bar{s}>1$ which increase to $s$, we may repeat this argument inductively to see that we can obtain $(*)-(* * *)$ for $r>r_{k}$, with $r_{k}$ defined inductively from the initial value $r_{0}=\infty$ by $\frac{1}{r_{k+1}}=\frac{1}{r_{k}}+\frac{p}{2}\left(1-\frac{1}{r_{k}}\right)$. Since $r_{k} \downarrow 1$, we therefore have $(*)-(* * *)$ for all $r, s>1$.

Proof of the lemma. The result follows from the Littlewood-Paley argument in [15] once we prove an $l^{2}$-valued inequality in place of the one [15, (2.10)] which is not proven for the case $1<p<2$. To get this inequality, we first see that the hypotheses of the lemma gives

$$
\int\left(\sup _{j}\left|\sigma_{j} * f_{j}\right|\right)^{p} v^{1 / r_{0}} \leq C_{p, r_{0}} \int\left(\sup _{j}\left|f_{j}\right|\right)^{p}\left(M M^{|\widetilde{\sigma}|} M_{s} v\right)^{1 / r_{0}},
$$

which follows from $(* * *)$ and the inequality $\sup _{j}\left|\sigma_{j} * f_{j}\right| \leq M^{|\sigma|}\left(\sup _{j}\left|f_{j}\right|\right)$. We can also show the inequality

$$
\begin{aligned}
\int \sum_{j}\left|\sigma_{j} * f_{j}\right|^{p} v & \leq C_{p} \int \sum_{j}\left|f_{j}\right|^{p} M^{|\widetilde{\sigma}|} v \\
& \leq C_{p} \int \sum_{j}\left|f_{j}\right|^{p} M M^{|\widetilde{\sigma}|} M_{\bar{s}} v
\end{aligned}
$$

The second line of this inequality follows by simply enlarging the weight on the first line, and the first line follows once we show $\left\|\sigma_{j} * f\right\|_{L^{p}(v)} \leq C\|f\|_{L^{p}\left(M^{|\widetilde{\sigma}| v)}\right.}$ uniformly in $j$. This in turn is shown by interpolating between the corresponding bound at $p=1$ (which follows easily by duality), and the trivial bound $\left\|\sigma_{j} * f\right\|_{\infty} \leq C\|f\|_{\infty}$.

Now, we may view the operation sending $\left\{f_{j}\right\}_{j \in Z}$ into $\left\{\sigma_{j} * f_{j}\right\}_{j \in Z}$ as a linear operator on the space of sequence-valued functions, so that (1) and (2) are weighted $l^{q}$-valued inequalities for this operator, taking $q=\infty$ and $q=p$ 
respectively. When interpolating with change of measures between function spaces of this type, the spaces that result are again weighted $l^{q}$-valued function spaces, with $q$ being the corresponding interpolated value and with the weight being the same as arises in the interpolation of the corresponding scalar-valued weight spaces. This may by seen by a straightforward modification of the scalar interpolation argument in [17] and a more general statement of this interpolation property may be found in [18]. As a consequence of this kind of interpolation between (1) and (2), we obtain the weighted $l^{2}$-valued inequality

$$
\int\left\{\sum_{j}\left|\sigma_{j} * f_{j}\right|^{2}\right\}^{p / 2} v^{1 / r_{1}} \leq C \int\left\{\sum_{j}\left|f_{j}\right|^{2}\right\}^{p / 2}\left(M M^{\widetilde{\sigma} \mid} M_{\bar{s}} v\right)^{1 / r_{1}}
$$

for $r_{1}$ as given in the statement of the theorem.

We now use the Littlewood-Paley argument of [15], with the square inequality appearing there being replaced in the case $1<p<2$ by $(\mathrm{S})$. This will give the inequality

$$
\int\left|T^{\sigma} f\right|^{p} v^{1 / r_{1}} \leq C_{p, r, s_{1}, s_{2}} \int|f|^{p}\left\{M_{s_{2}}\left(M M^{|\widetilde{\sigma}|} M_{s} M_{s_{1}} v\right)^{1 / r_{1}}\right\}
$$

for any $s_{1}, s_{2}>1$. This is essentially $(*)$ except that the weight on the right is complicated by extra iterations of the operator $M_{s}$. We shall eliminate these extra iterations using the Coifman-Rochberg inequality, which says that whenever $0<\theta<1$,

$$
M\left\{(M v)^{\theta}\right\} \leq C_{\theta}(M v)^{\theta}
$$

for all locally integrable $v$, with constant independent of $v$. We first choose $s_{1}>s$ and $s_{2}<r_{1}$ in $\left(*^{\prime}\right)$, next we apply the Coifman-Rochberg inequality, and then we relabel exponents, and this gives us $(*)$ for the desired range, which completes the proof.

Note. Of the Fourier transform requirements (1.9), (1.10) of [15] which are placed upon the measures, the bound $(1.9(\mathrm{i}))$ and the the first bound in (1.10) typically arise as a consequence of a condition on the support of the measures. An alternative proof for all $p>1$ of the two-weight inequalities is sketched in $[20$, Note in the proof of Theorem 4] under the stronger assumption that this support condition holds.

Steve HofmanN

Department of Mathematics, University Of Missouri-Columbia, Columbia Missouri 65211

E-mail address: hofmann@math.missouri.edu

DAVID K. WATSON

Department of Mathematical Sciences, University of Missouri-Columbia, Rutgers UNIVERSITY-CAMDEN, NJ 08102

E-mail address: dwatson@zodiac.rutgers.edu 


\section{References}

[1] J. Bergh and J. Löfström, Interpolation spaces. An introduction, Grundlehren der Mathematischen Wissenschaften, No. 223. Springer-Verlag, Berlin-New York, 1976.

[2] A. P. Calderón and A. Zygmund, On singular integrals, Amer. J. Math. 78 (1956), 289-309.

[3] J. Chen, D. Fan, and Y. Ying, Singular integral operators on function spaces, J. Math. Anal. Appl. 276 (2002), no. 2, 691-708.

[4] _ Certain operators with rough singular kernels, Canad. J. Math. 55 (2003), no. $3,504-532$.

[5] J. Chen and C. Zhang, Boundedness of rough singular integral operators on the TriebelLizorkin spaces, J. Math. Anal. Appl. 337 (2008), 1048-1052.

[6] Q. Chen and Z. Zhang, Boundedness of a class of super singular integral operators and the associated commutators, Sci. China Ser. A 47 (2004), no. 6, 842-853.

[7] R. Coifman and R. Rochberg, Another characterization of BMO, Proc. Amer. Math. Soc. 79 (1980), no. 2, 249-254.

[8] A. Cordoba and C. Fefferman, A weighted norm inequality for singular integrals, Studia Math. 57 (1976), no. 1, 97-101.

[9] J. Duoandikoetxea, Weighted norm inequalities for homogeneous singular integrals, Trans. Amer. Math. Soc. 336 (1993), no. 2, 869-880.

[10] J. Duoandikoetxea and J. L. Rubio de Francia, Maximal and singular integral operators via Fourier transform estimates, Invent. Math. 84 (1986), no. 3, 541-561.

[11] C. Fefferman and E. M. Stein, Some maximal inequalities, Amer. J. Math. 93 (1971), $107-115$.

[12] M. Frazier, B. Jawerth, and G. Weiss, Littlewood-Paley Theory and the Study of Function Spaces, CBMS Regional Conference Series in Mathematics, 79. Published for the Conference Board of the Mathematical Sciences, Washington, DC; by the American Mathematical Society, Providence, RI, 1991.

[13] J. García-Cuerva and J. L. Rubio de Francia, Weighted Norm Inequalities and Related Topics, North-Holland Mathematics Studies, 116. Notas de Matematica [Mathematical Notes], 104. North-Holland Publishing Co., Amsterdam, 1985.

[14] L. Grafakos and A. Stefanov, $L^{p}$ bounds for singular integrals and maximal singular integrals with rough kernels, Indiana Univ. Math. J. 47 (1998), no. 2, 455-469.

[15] S. Hofmann, Weighted norm inequalities and vector valued inequalities for certain rough operators, Indiana Univ. Math. J. 42 (1993), no. 1, 1-14.

[16] J. L. Rubio de Francia, F. J. Ruiz, and J. L. Torrea, Calderón-Zygmund theory for operator-valued kernels, Adv. in Math. 62 (1986), no. 1, 7-48.

[17] E. M. Stein and G. Weiss, Interpolation of operators with change of measures, Trans. Amer. Math. Soc. 87 (1958), 159-172.

[18] H. Triebel, Interpolation Theory, Function Spaces, Differential Operators, NorthHolland Mathematical Library, 18. North-Holland Publishing Co., Amsterdam-New York, 1978.

[19] A. M. Vargas, Weighted weak type $(1,1)$ bounds for rough operators, J. London Math. Soc. 54 (1996), no. 2, 297-310.

[20] D. K. Watson, Vector-valued inequalities, factorization, and extrapolation for a family of rough operators, J. Funct. Anal. 121 (1994), no. 2, 389-415.

College of SCIEnce

Hangzhou Dianzi University

Hangzhou 310018, P. R. China

E-mail address: purezhang@hdu.edu.cn 